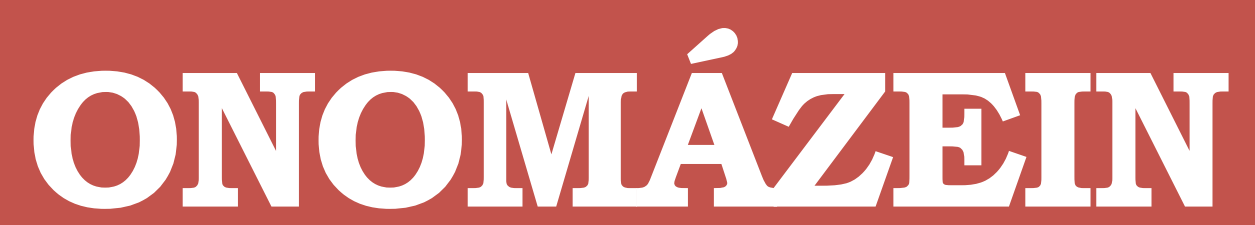

Revista de lingüística, filología y traducción
PONTIFICIA UNIVERSIDAD CATÓLICA DE CHILE FACUITAD DE LETRAS

\title{
Permanencia, innovación y obsolescencia en el repertorio antroponímico español
}

Permanence, innovation and obsolescence

in the Spanish anthroponymic repertoire

\section{Carmen Fernández Juncal}

Universidad de Salamanca

España

ONOMÁZEIN 53 (septiembre de 2021): 106-130

DOI: 10.7764/onomazein.53.02

ISSN: 0718-5758

\section{(c) $($ i) $\ominus$}

Carmen Fernández Juncal: Departamento de Lengua Española, Universidad de Salamanca, España.

| E-mail: cjuncal@usal.es 


\section{Resumen}

La presente investigación indaga acerca de la vitalidad de los antropónimos de la población residente en España comparando los usos onomásticos de las diferentes generaciones en términos de permanencia, innovación y obsolescencia. Apoyándonos en los datos proporcionados por el Instituto Nacional de Estadística (INE) y complementados por los inventarios de Mateos y Tucker (2008) y Fernández Juncal y Hernández Muñoz (2019) y el corpus SUBTLex, se repasan las características de los nombres estables del repertorio y su posición de referencia onomástica a lo largo de diversas generaciones. Asimismo, se especifican los diversos procedimientos de renovación del inventario, que agrupamos en cinco tipos de recursos: desde los préstamos hasta los cambios ortográficos, en funcionalidad y rango pasando por el papel de los compuestos y de las modas y tendencias. Finalmente analizamos la naturaleza de los nombres en declive y la presencia en este grupo de elementos idiosincrásicos. De manera transversal se señala el papel de la variable género, que muestra diferencias en los hábitos onomásticos dependiendo del sexo de su usuario.

Palabras clave: socionomástica; socioantroponimia; antropónimos; identidad social.

\section{Abstract}

This research investigates the vitality of the anthroponyms used by residents in Spain by comparing the onomastic uses of different generations in terms of permanence, innovation and obsolescence. Based on data provided by the National Statistics Institute (INE) and complemented by the inventories of Mateos and Tucker (2008) and Fernández Juncal and Hernández Muñoz (2019) and the SUBTLex corpus, the characteristics of the stable names in the repertoire and their onomastic reference position over several generations are reviewed. We also specify the various inventory renewal procedures, which we group into five types of resources: Ioans, orthographic changes, changes in functionality and range, the role of compounds and fashions and trends. Finally, we analyze the nature of declining names and the presence in this group of idiosyncratic elements. The role of the gender variable is pointed out transversally, showing differences in onomastic habits depending on the sex of the user.

Keywords: socio-onomastics; socioanthroponymy; anthroponyms; social identity. 


\section{Los estudios socionomásticos y los modelos atributivos nominales}

El nombre propio y muy especialmente los nombres que designan a personas o antropónimos tienen un comportamiento muy específico dentro de la clase nominal, no solo desde el punto de vista de la gramáticaㄹ, sino también en otros ámbitos muy ligados a los usos sociales y el entorno cultural. De hecho, como vimos en Fernández Juncal (2011), el sistema de antropónimos es una parcela léxica muy afectada por las variaciones que conforman el diasistema de la lengua: por su implantación geográfica; por su evolución diacrónica; por otras variables de tipo diastrático como el género, la edad o el nivel socioeconómico, y por su variación contextual o diafásica a través del uso de hipocorísticos y otras designaciones como los apodos y los pseudónimos.

El uso de nombres para identificar personas y lugares es un universal lingüístico (Hough, 2016); en este sentido constituyen un aspecto esencial de la comunicación humana permitiendo la distinción entre individuos, pero, como todos los elementos de las diferentes lenguas, son muestra genuina de los sistemas de los que emanan, por lo que acomodan su estructura formal y su funcionamiento a las reglas internas de cada variedad (Wheeler, 2018), pero también al conjunto de actitudes, valores y principios que rigen en cada sociedad y cultura.

En este aspecto se insertan los estudios socionomásticos, de todavía corta trayectoria y con mirada interdisciplinar (Seide, 2016), ya que se centra en un campo de estudio de mucho interés para otros especialistas, como los sociólogos, que ven en la onomástica un área de prueba del desarrollo de conductas y creencias colectivas. La socionomástica, considerada la vertiente sociolingüística de la onomástica (Ainiala y Östman, 2017), concentra su atención en una serie de tareas (Bramwell, 2014): determinar los nombres empleados en cada comunidad y examinar los rasgos comunes en todas ellas, averiguar las motivaciones que originan la elección de los nombres y fijar la diversidad de actitudes y valores que subyacen en este proceso en el conjunto de las comunidades (Ainiala y Östman, 2017; Aldrin, 2016 y 2017).

Elegir un nombre rara vez se limita a un mecanismo de etiquetado, al menos en las sociedades occidentales. A menudo es un proceso largo y complejo, al que los progenitores del recién nacido dedican tiempo e interés. Se toman en consideración, de manera consciente o inconsciente, muchos aspectos a la vista de sus posturas emocionales, estéticas, ideológicas y sociales, así como sus experiencias personales y los deseos de futuro para su hijo (Aldrin, 2016 y 2017). No se trata, por lo tanto, de un mero trámite en el que intervenga el azar, sino un acto social en el que se atienden (o se rechazan) Ias normas sociales o costumbres de tipo etnocultural establecidas durante generaciones. Los padres se comprometen con esta acción

1 Sobre el comportamiento morfosintáctico de esta clase de palabras son ya trabajos de referencia los de Gary-Prieur (1994), Bajo Pérez (2002) y Fernández Leborans (1999). 
para expresar su propia identidad y la del futuro adulto, influidos, según Aldrin (2011, apud Jiménez Segura, 2014), más por la red social en la que se insertan que por otras variables como su edad, profesión, etc., sometidos a una tensión entre lo individual y lo común. Es lo que Offroy ha llamado el proyecto parental y Besnard y Desplanques (apud Jiménez Segura, 2014) explican de la siguiente manera: el hablante no se fija tanto en los modelos que emanan de su grupo como en los del grupo al que aspira a pertenecer; al tiempo, busca un nombre diferenciador pero no extravagante respecto a la norma del lugar o la época. De hecho, Colman y otros (1981) señalan la importancia de la familiaridad como uno de los elementos decisivos a la hora de optar por un nombre u otro, de forma que los nombres más populares suelen ser más apreciados en detrimento de los menos comunes. Por otra parte, el proceso global se desarrolla en una serie de condiciones de fuerzas opuestas. Así, Kessler y otros (2012) explican esta dinámica en los siguientes términos: hay una fase de crecimiento de frecuencia del nombre, que refleja una especie de proceso de infección $n^{2}$ en las redes sociales de los electores, que buscan un nombre original y atractivo. El nombre se convierte en popular y luego en demasiado popular para satisfacer los deseos de individualidad de los progenitores, por lo que su interés decae. Según Colman y otros (1981), este principio de la U invertida, que es extensible a otros fenómenos ligados a la moda (colores, ropa, etc.), explica cómo, una vez un elemento adquiere su nivel crítico de familiaridad, llega a su pico de popularidad y empieza a declinar en atractivo.

Los modelos atributivos han variado a lo largo del tiempo en distintas sociedades. De acuerdo con Aldrin (2011, apud Jiménez Segura, 2014), hay varias tendencias que definen el desarrollo antroponímico de las últimas décadas: la preferencia por nombres de carácter religioso da paso a una fase de menor uso de este tipo de nombres, incrementando el número de elementos del repertorio (Frai, 2016; Jiménez Segura, 2014), con importancia decisiva del factor moda y menor conservadurismo para el caso de los nombres femeninos. López Franco (2014), que encuentra muchas similitudes entre el modelo mexicano y el modelo francés, señala además el papel decisivo de los medios de comunicación, no tanto como creadores de tendencias cuanto como difusores de ellas, y también la relativa importancia de los préstamos lingüísticos en la atribución de los nombres de pila, especialmente a finales del siglo XX. Según Vandebosch (1998), los medios de comunicación cumplen con tres funciones en el ámbito onomástico: aportar nuevos nombres a la atención del público, individualizar las asociaciones que pudiera tener un nombre y contribuir a la creación de estereotipos en torno a ciertos nombres.

2 De hecho, estos autores relacionan esta etapa de desarrollo con la que siguen las epidemias.

3 En un nivel más concreto García Gallarín (2007b) señala cuatro etapas en el desarrollo onomástico madrileño: 1. a. desde 1900 hasta 1940, que denomina de "las dos Españas"; 2. a. desde 1939 hasta 1975 (etapa franquista); 3. a.: 1976 - finales del siglo XX (consolidación de la democracia), y 4.a. desde 1996. 
Jiménez Segura (2014) clasifica los modelos atributivos en tres: el modelo de atribución tradicional incluye antropónimos ligados al ámbito religioso y también a la herencia dentro del ámbito familiar. El modelo de atribución por moda se refiere a los nombres que se encuentran presentes en la norma antroponímica durante un tiempo motivada fundamentalmente por imitar la denominación de personas famosas o personajes en boga. Frai (2016) incluye también en este apartado razones estéticas: gusto por la sonoridad, la pronunciación e incluso la escritura del nuevo nombre. Finalmente los modelos de atribución libre comprenden los casos no mencionados, que Frai (2016) detalla en los siguientes: influencia histórica o política, circunstancias de tiempo y lugar del nacimiento, creencias individuales, originalidad, causalidad, significado del nombre, disponibilidad, influencia literaria y etnia.

\section{Marco normativo}

La atribución nominal permite una gran libertad a los electores ${ }^{4}$, pero en cada comunidad existen normas que restringen, en mayor o menor medida, esa capacidad de selección (BuIliet, 1978: 489): "One of the few predictable opportunities for the exercise of free will that comes the way of most human beings is the bestowal of names upon their children. To be sure, local or national custom may legally restrict or otherwise limit the scope of that freedom in some cases; but by and large, there is normally some choice to be made, and the beneficiary of the choice, the child, is inevitably powerless to influence it".

El periodo que nos ocupa, desde comienzos del siglo XX hasta nuestros días, ha visto desarrollarse diferentes sistemas políticos, acontecimientos históricos que han cambiado la evolución de España, que han venido acompañados en paralelo con la evolución del entorno legislativo respecto a la asignación de nombres para los recién nacidos, y que ha tenido repercusiones en la amplitud de la nómina de nombres de pila.

Siguiendo la cronología que nos propone Fernández Pérez (2015), se han sucedido cuatro hitos en ese marco normativo, formado por leyes de diferente rango: en primer lugar, la Ley Provisional de Registro Civil de 1870, que se puede considerar la primera reguladora, pero que en el fondo no deja de ser una mera secularización del registro parroquial, que hasta ese momento había servido como depositario del acto de inscripción de los recién nacidos. En segundo lugar, la Ley de 8 de junio de 1957, sobre Registro Civil, rigió los hábitos y prácticas de las décadas siguientes hasta la llegada de la democracia. En tercer lugar, la promulgación de la Constitución Española de 1978 supuso cambios sustanciales en algunas de las restricciones acordadas en la ley franquista. En cuarto y último lugar, contamos en la actualidad con la Ley

4 En términos de García Gallarín (2007b). 
20/2011, de 21 de julio, del Registro Civil, cuyo desarrollo íntegro se completará en junio de 2020. Esta última normativa casi no supone restricciones en la elección de nombre, apenas tres: la limitación de poseer como máximo dos nombres simples o uno compuesto, que los nombres no sean contrarios a la dignidad de la persona ni hagan confusa la identificación, y que el nombre elegido no sea el mismo que se impuso a un hermano con los mismos apellidos, salvo que hubiera fallecido.

Estos tres impedimentos habían sido compartidos, con diferencias de matiz, por las normas precedentes, pero la ley franquista, precedida por otras normativas de rango inferior, puso además trabas que afectaron en gran medida a los usos antroponímicos de la época, de las que destacaremos dos. Por una parte, se establece que "en la inscripción se expresará el nombre que se dé al nacido, que debe ser, en su caso, el que se imponga en el bautismo" (párrafo primero de la ley de 8 de junio de 1957 de Registro Civil). En paralelo, el canon 761 del Código de Derecho Canónico de 1917 estaba formulado en los siguientes términos: "Procuren los párrocos se imponga un nombre cristiano y, de no conseguirlo, añadan el nombre de algún santo al escogido por los padres y consignen ambos en el libro de bautizados"5. La aplicación simultánea de ambas normativas implica, por una parte, el empleo mayoritario del santoral o las advocaciones marianas como catálogo de referencia para los padres y, por otra parte, la cristianización de los nombres mediante diversos procedimientos, el más habitual, la inclusión de nombres cristianos reconocidos, principalmente María y José, que se añadían, generalmente antepuestos, pero también pospuestos, a los elegidos por los padres.

Por otra parte, desde finales de la guerra civil7 se prohíbe para los ciudadanos españoles el uso de nombres que no sean castellanos, lo cual excluye del inventario nombres de lenguas extranjeras y también nombres del resto de las lenguas peninsulares.

La promulgación de la Constitución de 1978 permitió abolir estas y otras restricciones y de esa forma se dio entrada a nombres de todo tipo de procedencias, de lenguas extranjeras y en particular de las lenguas vernáculas de las diferentes comunidades españolas.

5 En el Código de Derecho Canónico vigente, de 1983, el canon 855 tiene un tono más de indicación o sugerencia: "Procuren los padres, los padrinos y el párroco que no se imponga un nombre ajeno al sentir cristiano".

6 Aunque no tuviera refrendo legal, el empleo de María como apoyo de nombre de advocaciones no data de esta época; García Gallarín (2009 y 2014a) lo atestigua en el paso del periodo medieval al clásico. Se ha consultado a este respecto con diversas fuentes eclesiásticas. Parece que el empleo de María como apoyo nominal se extendió, además de los nombres no cristianos, también a nombres de advocaciones marianas y, en general a todo tipo de nombres.

7 En la orden de mayo de 1938 y en la propia ley de 1957. 


\section{Fuentes y otras cuestiones metodológicas}

Según Guérios (1981), existen primordialmente dos maneras de llevar a cabo un estudio antroponímico: desde el punto de vista de su etimología, es decir, examinando su origen, o desde el punto de vista social. En esta investigación adoptaremos el segundo punto de vista, por lo que tendremos que recurrir a bases de datos que recojan información de colectivos o comunidades de cierta extensión.

El presente estudio tiene como fuente primaria parte de los datos que el Instituto Nacional de Estadística (INE) ${ }^{8}$ hace periódicamente públicos en su web institucional, para nuestro caso los publicados el 29 de mayo de 2018 actualizados a 1 de enero de 2017. Se trata de los siguientes9:

- Nombres más frecuentes entre personas residentes en España con frecuencia igual o superior a 20 (se especifica en este caso la edad media de los portadores, dato muy significativo para nuestros intereses ${ }^{10}$.

- Los 100 nombres más frecuentes para todo el territorio nacional con dos variantes: una que incluye los nombres compuestos y otra que los excluye, considerando sus componentes como unidades simples.

- Los 50 nombres más habituales por década de nacimiento desde antes de los años 30 hasta 2016.

- Los 100-150 nombres más frecuentes entre los recién nacidos desde 2002 hasta 2016.

Los datos se obtienen a partir de la estadística de Padrón Continuo, base de datos que se actualiza con los ficheros recibidos desde los distintos ayuntamientos, bajo la coordinación del propio INE. El fichero final ha sido tratado garantizando que los datos sean homogéneos y para que las variables que se difunden tengan valores correctos.

La información onomástica que presenta este organismo es completa con algunas restricciones. En primer lugar, están excluidos de las listas los nombres con frecuencia inferior a 20. Se trata de una práctica habitual en los registros institucionales de todo el mundo (con diferencias en los límites), que evita atentar contra el derecho a la privacidad. Esta norma nos impide acceder a nombres únicos, con un solo poseedor, que podría tener su interés a pesar de no ser significati-

8 García Gallarín (2014a: 19) afirma que se trata de una fuente que "permite investigar con rigor las tendencias contemporáneas de la antroponimia hispánica”.

9 Además de los expuestos, el INE publica los 50 nombres más frecuentes por provincia y los 20 más frecuentes por nacionalidad. Cuenta asimismo con una aplicación para consultas específicas acerca de nombres concretos.

10 No obstante, no debemos perder de vista que, como señala Weitman (1981), el análisis y las inferencias sobre los usos antroponímicos deben referirse a los electores de nombres y no a sus portadores. 
vos estadísticamente. Por otra parte, en estos registros, no hay información de tipo sociológico ni onomástico sobre el individuo portador y sobre su entorno familiar (padres, abuelos, etc. ${ }^{11}$.

Weitman (1981) se pregunta acerca de la idoneidad de manejar en los estudios onomásticos el conjunto del corpus o solo una parte representativa. En nuestro caso, la propia configuración del repertorio y los objetivos perseguidos nos proporcionan la respuesta: utilizaremos de manera primordial los datos seleccionados de acuerdo con las frecuencias más elevadas, es decir, la popularidad de los diferentes nombres, que representan de forma más ajustada las tendencias generales; en algunas ocasiones manejaremos los datos globales, y en otras utilizaremos como referencia ambos listados de manera adicional y contrastiva.

Complementamos esta gran base de datos con otros recursos secundarios, como el corpus elaborado por Mateos y Tucker (2008), basado en un directorio telefónico, o los catálogos de nombres propios disponibles recabados por Fernández Juncal y Hernández Muñoz (2019) en una encuesta, realizada en 2018 entre hablantes de la modalidad centro-peninsular. Además tomaremos también como referencia la frecuencia de los nombres propios que aparecen en SUBTLex. Se trata de un listado de frecuencias, basado en el análisis de un corpus en torno a 40 millones de palabras, procedentes de los subtítulos de películas. Se considera que este tipo de corpus predice de manera más ajustada la variación léxica que otras fuentes escritas de más extensión, precisamente porque están basados en textos orales.

En cualquier caso, los datos primarios cumplen todos los requisitos que enunciaban Bloothooft y Mandemakers (2014) para garantizar la calidad de las diversas fuentes: no necesitan permisos de acceso especiales, están digitalizados y, sobre todo, son representativos y permiten análisis cuantitativos fiables.

Respetamos y no unificamos las variantes que el INE inventaría en el corpus, que son muchas y muy variadas (como ocurre con Ainhoa, Aynoha, Ahinoa, Ainoha o Ainoa) ${ }^{12}$. Asumimos esta decisión porque son reproducción fiel de los nombres registrados en el padrón y porque en muchas ocasiones no está claro el nombre de referencia. Además, como advertimos, las variantes por debajo de 20 portadores ya han sido excluidas de las listas.

Respecto a los estadísticos empleados, aportaremos los tres elementos que Weitman (1981) considera básicos en este tipo de trabajos: frecuencias absolutas en crudo, frecuencias pro-

11 Estos datos sí se encuentran en los registros civiles, pero los estudios deben estar entonces enfocados a comunidades concretas, más pequeñas, porque, como señalan Bloothooft y Mandemakers (2014), no suelen estar digitalizados en su faceta histórica y además presentan problemas de salvaguarda de la privacidad.

12 Por otra parte, en el proceso de depuración, el INE elimina las tildes y las diéresis y conserva el apóstrofo y la cedilla. 
porcionales a la población afectada y rango de los diferentes nombres respecto a los demás en la lista de frecuencias. El indicativo de menor utilidad es el primero porque en muchas ocasiones los cambios en sus valores no reflejan tanto modificaciones onomásticas como modificaciones en las dimensiones de la población. El último valor, el rango, es la medida que mejor sirve para trazar los movimientos en la popularidad de los nombres ${ }^{13}$.

Este mismo autor señala los aspectos que un examen onomástico debería abordar: la denotación y connotación de los nombres, las características morfológicas y fonéticas, así como su origen y su comparación con otros nombres extranjeros homónimos. Este análisis parece diseñado para nombres aislados o agrupaciones concretas de nombres, pero intentaremos, dentro de las limitaciones de esta investigación, pasar revista a alguno de estos puntos aplicados a conjuntos más amplios de antropónimos.

\section{Análisis de resultados}

\subsection{La permanencia onomástica}

Antes de precisar los cambios que se producen en el repertorio, nos detendremos brevemente en los nombres que permanecen en las 50 primeras posiciones a lo largo de todo el periodo estudiado. En los gráficos la y lb se observa la evolución de los nombres de hombre y de mujer que cumplen este requisito:

\section{GRÁFICO IA}

Evolución de nombres masculinos que se mantienen en las primeras 50 posiciones (antes de los 30-2016)

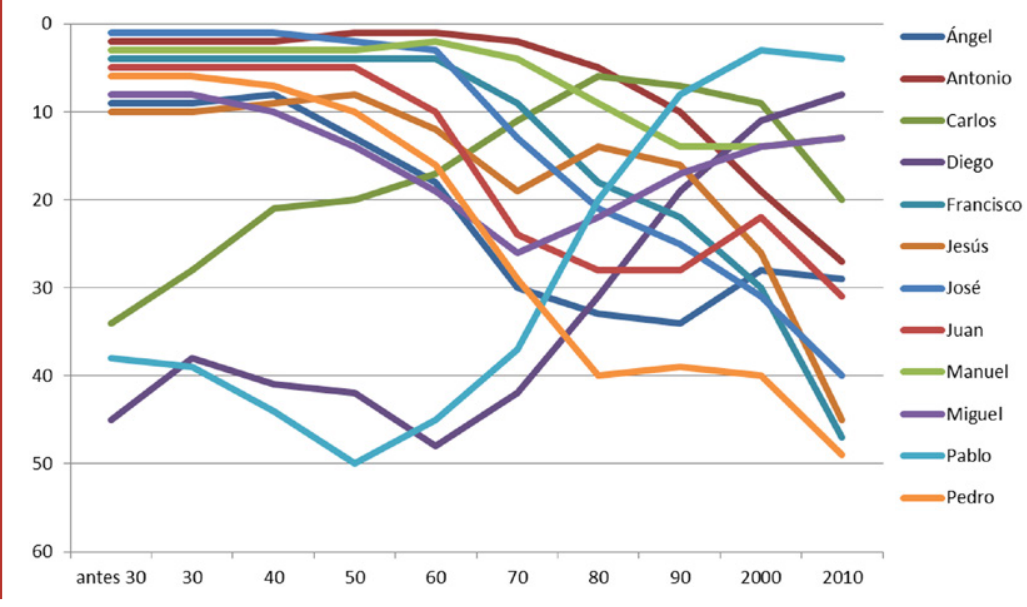

13 Por ejemplo, Lieberson (2000), para su trabajo, que podemos considerar de referencia, analiza los nombres más frecuentes (los 10 y los 50 primeros en periodos de tiempo desde 2 hasta 100 años). 


\section{GRÁFICO 1B}

Evolución de nombres femeninos que se mantienen en las primeras 50 posiciones (antes de los 30-2016)

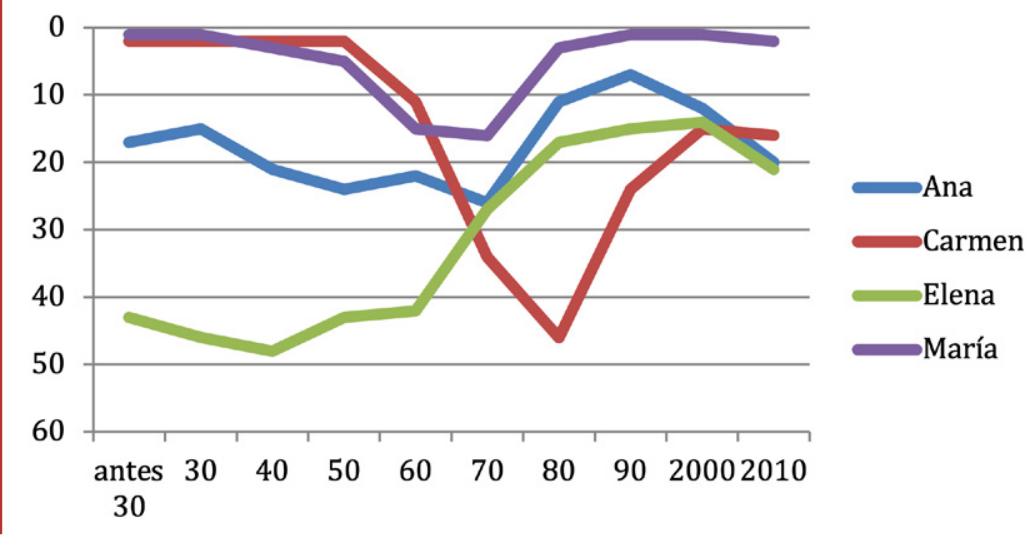

En primer lugar, el número de nombres de varón que han resistido el empuje de otros nuevos triplica al de mujeres. Además, el 60\% de ellos comparte las primeras 10 posiciones (acaparando una frecuencia acumulada muy elevada, como vimos en Fernández Juncal (2019), para luego descender en sus posiciones en la época actual. Al tiempo, nombres menos populares en el principio del periodo (Pablo, Diego o Carlos) han alcanzado puestos superiores en épocas más recientes. En el caso de las mujeres, los nombres poseen más dispersión, exceptuando el constante María, que apenas baja puestos, únicamente en la época en que es más empleado como parte de los compuestos femeninos. Como veremos más adelante, en el ámbito español, para los hombres ha funcionado de manera más constante el sistema de herencia de nombres y el conservadurismo onomástico en general mientras que para las mujeres han primado otros valores, como la unicidad y la originalidad (Fernández Juncal, 2002, 2009 y 2018; García Gallarín, 2007b), propensión que también se confirma en otras diatopías (Rossi, 1965; Lieberson, 2000, o López Franco, 2014).

En la tabla 1 se ven las posiciones que ocupan los nombres permanentes en los diferentes corpus (véase la tabla en la página siguiente).

Como comprobamos, todos los nombres que han permanecido en el conjunto de los 50 nombres más empleados por décadas del corpus del INE aparecen en posiciones relevantes en los otros dos repertorios de los que disponemos y poseen frecuencias altas en el corpus textual SUBTLex. Así pues, los nombres más estables en el conjunto de los antropónimos del corpus INE tienen también relevancia en investigaciones que parten de fuentes distintas. Es el caso de Mateos y Tucker (2008), que, como dijimos, está basado en listines telefónicos ${ }^{14}$. Sin em-

14 En la interpretación de los datos hay que considerar la menor presencia de mujeres en el corpus por ser menor el número de titulares de teléfono de esa condición. Además, como advertíamos, 


\section{TABLA 1}

Presencia de los nombres permanentes en los diferentes corpus

\begin{tabular}{|c|c|c|c|c|c|c|}
\hline & & $\begin{array}{l}\text { POSICIÓN } \\
\text { INE } 2017\end{array}$ & $\begin{array}{l}\text { EDAD } \\
\text { MEDIA } \\
\text { (INE) }\end{array}$ & $\begin{array}{c}\text { POSICIÓN } \\
\text { CORPUS } \\
\text { MATEOS-TUCKER }\end{array}$ & $\begin{array}{l}\text { POSICIÓN } \\
\text { CORPUS DL }\end{array}$ & $\begin{array}{l}\text { FRECUENCIA } \\
\text { SUBTLEX }\end{array}$ \\
\hline \multicolumn{7}{|l|}{ Varón } \\
\hline & Ángel & 20 & 49,9 & 11 & 5 & 31,18 \\
\hline & Antonio & 1 & 55,5 & 2 & 3 & 21,66 \\
\hline & Carlos & 8 & 38,3 & 23 & 8 & 39,33 \\
\hline & Diego & 31 & 31,8 & 71 & 31 & 15,58 \\
\hline & Francisco & 4 & 56,9 & 4 & 6 & 21,47 \\
\hline & Jesús & 12 & 48,0 & 8 & 13 & 86,88 \\
\hline & José & 2 & 60,8 & 1 & 1 & 20,96 \\
\hline & Juan & 6 & 55,4 & 5 & 2 & 26,88 \\
\hline & Manuel & 3 & 54,6 & 3 & 9 & 10,72 \\
\hline & Miguel & 15 & 46,2 & 13 & 18 & 13,68 \\
\hline & Pablo & 21 & 26,7 & 61 & 10 & 11,59 \\
\hline & Pedro & 18 & 53,1 & 6 & 7 & 14,5 \\
\hline \multicolumn{7}{|l|}{ Mujer } \\
\hline & Ana & 11 & 43,6 & 45 & 2 & 17,52 \\
\hline & Carmen & 3 & 60,4 & 10 & 3 & 16,42 \\
\hline & Elena & 24 & 36,3 & 95 & 8 & 15,07 \\
\hline & María & 2 & 48,7 & 9 & 1 & 49,73 \\
\hline
\end{tabular}

bargo no existe correlación estadística entre las posiciones del INE y las frecuencias de las listas SUBTLex, debido a la propia configuración de estas últimas, que agrupan y, por lo tanto, distorsionan las frecuencias de homónimos (como ocurre con ángel y Ángel) o de términos que pueden tener un valor añadido (como ocurre con Jesús, que se emplea también como exclamación o como parte de unidades léxicas más complejas).

los datos no están desglosados por sexo. No obstante, el índice de correlación de Pearson entre Ios valores de INE y Mateos y Tucker $(2008)$ es muy elevado, tanto para hombres $(0,77)$ como para mujeres $(0,99)$. Recordemos que este índice se mueve en un rango máximo entre 1 (para relaciones directas) y-1 (para relaciones inversas) y que o es el valor de correlación nula. 
Sí hay gran concordancia entre los resultados del INE y los obtenidos en el estudio (Fernández Juncal y Hernández Muñoz, 2019), sobre los nombres de persona disponibles ${ }^{15}$. La disponibilidad léxica, como metodología léxico-estadística, permite detectar el conocimiento del mundo compartido por los individuos y la existencia de elementos ejemplares dentro de una categoría. Para el caso de los nombres permanentes se refrenda la posición de estereotipo que estos nombres poseen como términos recurrentes en el lexicón de los hablantes cuando se les somete a este estímulo.

\subsection{La renovación onomástica}

En este apartado analizaremos la renovación del catálogo de nombres de pila en España basándonos en dos tipos de datos. Por una parte, separaremos de los nombres totales aquellos que tienen una edad media inferior a 20 años; es decir, los nombres cuyos usuarios son mayoritariamente jóvenes. Por otra parte, contaremos con las listas de los nombres más populares elegidos para los recién nacidos entre 2002 y 2016. En la tabla 2 se detallan las dimensiones del corpus del que partimos.

\section{TABLA 2}

Detalle del corpus de nombres con edad media inferior a 20 años

\begin{tabular}{lcc} 
& HOMBRES & MUJERES \\
Número total de nombres & 24.601 & 25.091 \\
\hline Nombres con edad media inferior a 20 años & $2788(11,3 \%)$ & $2857(11,4 \%)$ \\
\hline Nombres con edad media inferior a 20 años con más de 5000 portadores & 51 & 68 \\
\hline Nombres compuestos & $1194(42,8 \%)$ & $898(31,4 \%)$ \\
\hline
\end{tabular}

El número de nombres con edad media inferior a 20 años representa poco más de una décima parte del total, lo que es esperable considerando que la mayoría de los nombres, los más comunes, se concentran en las edades intermedias, demográficamente más pobladas.

\subsubsection{La renovación del sistema antroponímico: factores internos y externos}

Antes de detallar los procedimientos de innovación onomástica, es necesario hacer constar la diversidad de factores que han originado cambios en el repertorio, tanto en la incorporación de nuevas unidades como en el aumento o disminución de frecuencias en otras. Sin duda, son los movimientos demográficos los principales causantes de esas modificaciones detectables

15 En este caso el índice de correlación de Pearson también es muy notable para los nombres de varón $(0,73)$ y para los de mujer $(0,9)$. 
desde antiguo (García Gallarín, 2007a, 2007b, 2010 y 2014a). En la actualidad, no es difícil comprobar la influencia de fenómenos como la extensión de los medios de comunicación, el cambio de valores estéticos, la traslación del concepto de prestigio, la mejora del conocimiento idiomático, la evolución en los vínculos familiares o la secularización social (García Gallarín, 1997). No obstante y como ya advertíamos, es la inmigración ${ }^{16}$ (y su consecuente contacto con otras lenguas y otros modelos onomásticos) la que produce mayores alteraciones en el catálogo en procesos que, como ocurre con el caso español, son de ida y vuelta (García Gallarín, 2007a y 2014a). Así, el repertorio hispanoamericano, aunque con el paso de los años ha ido distanciándose de las tendencias peninsulares ${ }^{17}$, tiene una gran base española y a su vez ha influido, como veremos, en algunos de los procesos detectados en España.

A partir de los datos representados en la tabla 2, llevaremos a cabo un análisis de tipo cualitativo que nos permita detectar los procedimientos de innovación onomástica que se han puesto en marcha en las últimas generaciones ${ }^{18}$. Como veremos, no se trata en todos los casos de mecanismos que no se hayan empleado con anterioridad, pero sí que se aplican de manera especialmente intensa y que con su uso incorporan elementos nuevos.

\section{A. Préstamos}

A1. El primer recurso de ampliación del repertorio lo constituyen los extranjerismos. Estos son de tipo muy variado y de muy diversa procedencia: además de los esperables y abundantes en inglés (Brandon, Michael, Elliot, Scarlett, Ashley, Nicole, Chelsea, Hillary), hay nombres de otras procedencias, como resultado de la globalización y de los movimientos de población en inmigraciones, así como las tendencias instaladas en algunos colectivos de este carácter ${ }^{19}$ : italiano (Gioele, Angelo, Allegra), francés (Amelie, Giselle), árabe (Luqman, Abdalah, Arwa, Nourhan), chino (Yixiang, Jinxuan, Ziqi), rumanos (Ionut, Rares), panyabí (Gurkirat, Dilpreet), polaco (Kacper), hawaiano (Kai, Vaitiare), ruso (Nikita), japonés (Akira, Ao, Sakura, Aiko), ucraniano (Yehor, Matviy), lituano (Dovydas, Eimantas, Ugné), quechua (Amaru), escandinavo (Viggo). Para

16 Según datos del INE, a I de enero de 2019 la cifra de inmigrantes en España llegó a 4,8 millones (más del 10\% de la población total), dato que se acerca a los 5,4 millones que llegaron a residir entre 2009 y 2010.

17 De acuerdo con García Gallarín (2010), las discrepancias se deben fundamentalmente al influjo de la oralidad en al escritura, la oficialización de hipocorísticos y la introducción de préstamos además de la mayor libertad americana en el empleo de nombres dobles o múltiples y en la composición.

18 García Gallarín (2014a) señala cómo el interés por la identificación acrecienta la complejidad del sistema de denominación personal. En García Gallarín (2014b) analiza los procedimientos que se han desarrollado para evitar la ambigüedad.

19 Es lo que ocurre con el colectivo inmigrante hispanoamericano, más proclive a admitir nombres de otros orígenes (García Gallarín, 2007a). 
los préstamos del inglés podemos encontrar casos paralelos con la situación que describe y clasifica Thonus (1992) para Curitibá, en Paraná. Podemos distinguir en nuestro corpus los siguientes tipos: idénticos a los nombres ingleses de uso corriente (Bradley, Clark, Brittany, Phoebe), nombres ingleses con grafía en español (Neizan, Dilan, Braian, Estiven, Nicol, Dayana), españoles con apariencia inglesa (Matheo, Mayka, Aithana, Eryka, Karlota, Koral, Nathalia) e ingleses con falsa escritura inglesa (Uhon, Kevyn, Maykol, Keisy, Kaili, Kloe, Mishell).

A2. Otro recurso de innovación es la incorporación de nombres procedentes de las otras lenguas peninsulares: gallego (Brais, Xenxo, Antía, Uxía), catalán (Llura, Llum, Oleguer) y vasco (Peio, Xare, Illari, Itzea), y muy numerosos de la tradición guanche (Mencey, Nareme, Aithamy, Ubay, Agoney, Yamiley, Yahiza). Los datos que aporta Cid Abasolo (2007) y los que manejamos en Fernández Juncal y Hernández Muñoz (2019) nos hacen pensar que no es una tendencia localizada en las comunidades lingüísticas donde se emplean esas variedades, sino que se han extendido al resto del territorio con gran éxito.

\section{B. Nombres compuestos}

El uso de nombres compuestos o múltiples ${ }^{20}$ ha sido puesto de manifiesto desde antiguo (García Gallarín, 2009), pero el empleo efectivo ha variado de intensidad a lo largo de los años. En Fernández Juncal (2019) detallamos la evolución en la utilización de este mecanismo a lo largo del siglo XX y lo asociamos a un proceso de transición entre el empleo de los nombres heredados frente a los nombres no heredados.

Pues bien, en nuestra investigación previa detectábamos que, como veremos más adelante, los nombres con edad media más elevada muestran cifras discretas en el porcentaje de nombres compuestos. Estos porcentajes van creciendo hasta alcanzar el máximo en los nombres de edades medias entre 50 y 60 años (82,4\% de los casos). A partir de esa época, las proporciones descienden paulatinamente hasta alcanzar el nivel medio de los jóvenes en la actualidad (ver tabla 2) ${ }^{21}$. Examinando la situación actual y acudiendo a una segunda fuente, la lista de los 100/150 primeros nombres de los recién nacidos desde 2002 hasta 2016, comprobamos el empleo cada

20 Este fenómeno posee varias denominaciones. García Gallarín (2014a) prefiere utilizar la denominación de doble o múltiple y reservar compuesto como término técnico para los que conforman una sola unidad morfológica producto de la fusión de dos o más simples. Como nuestro estudio no es de carácter etimológico, empleamos compuesto, que es el vocablo de uso común, también en repertorios y normativas.

21 Seide y Grespan (2013), que emplean el término doble (duplo), hallan, en un análisis diacrónico en Toledo-Paraná (Brasil), las mismas fluctuaciones con un repunte en la época actual, sobre todo en los casos de nombres no asentados. En su caso, encuentran más diversidad de nombres dobles para mujer que para varón, que presenta más nombres repetidos. La mayor parte de los compuestos de mujer se forman con el nombre María, tal como ocurre en nuestro estudio. 
vez más minoritario de los nombres compuestos, al menos de los asentados por la costumbre y la tradición. No obstante, sigue empleándose este mecanismo como forma de denominación, como corroboran los datos respecto a los nombres con edad media inferior a 20 años (42,8\%). Lo que ocurre ahora es que no estamos mayoritariamente ante un compuesto propiamente dicho, sino ante un nombre yuxtapuesto. Es esta la distinción que establecen algunos estudios como el de López Franco (2010) y el de Frai (2016), que diferencian entre los conjuntos únicos asentados por el uso (nombres compuestos) y los no asentados (nombres yuxtapuestos o múltiples)22. Así, los primeros proceden de bases tradicionales y son combinaciones estereotipadas Uosé Antonio, Francisco Javier, Juan Pedro, Ana Isabel), mientras que los segundos son el resultado de la adición de dos unidades, de nuevo o viejo cuño, no vinculadas de manera consuetudinaria (Samuel Alejandro, Sebastián Gabriel, Estela Carmen, Carla Victoria, Ana Luna). Estos últimos, al tratarse de creaciones más o menos particulares de los padres, tienen más dificultad para instalarse en los puestos altos de frecuencia, como los que usamos de referencia.

Son de destacar en este mismo apartado los muy numerosos nombres yuxtapuestos de carácter híbrido, que combinan nombres de diferentes procedencias: Steven Javier, Bryan Miguel, Giovanni Manuel, Iker Alejandro, Ainhoa Carmen, Nicole Alejandra. En parte, se satisface el deseo de originalidad mediante el empleo simultáneo de dos recursos de innovación y, por otra parte, se cumple con un deseo de integración de personas de origen diverso en la nueva comunidad de acogida (Raúl Mihai, Alejandro Ionut, Andreea Anamaría).

\section{Modas y tendencias}

Bush y otros (2018) distinguen entre novedades, modas y tendencias en función de su tiempo de impacto. En nuestro corpus la influencia de estos valores se manifiesta en dos vertientes. Por una parte, se recuperan nombres clásicos que apenas formaban parte del repertorio actual, como Platón, Eneas, Ayax, Zeus, Beltrán, Telmo, Dante, Lautaro, Juno, Elektra, Gadea, Ginebra, Vera, Gala o Galatea. Por otra parte, se incorporan nombres de nuevo cuño de origen externo en su mayoría, asociados a personas famosas y cuyo conocimiento procede primordialmente de los medios de comunicación²3: Keylor, Neymar, Lennon, Pablo Nicolás, Daenerys, Arya, Bryanna/Brianna, Bimba, Rihanna, Suri, Miley, Salma, Dakota, Sakira/Shakira o Malú. Como se observa, este fenómeno afecta especialmente a los nombres de mujer ${ }^{24}$.

22 La legislación actual distingue también entre dos nombres simples y uno compuesto.

23 A este respecto y aunque es difícil establecer una distinción clara, Brender (apud Thonus, 1992) llegó a distinguir entre unique and novel names (de padres instruidos) e idiosyncratic appellations (de padres menos instruidos o de clases sociales más bajas).

24 También es fenómeno que afecta al nivel sociocultural. Vandebosch (1998) señala que en los países europeos las clases educadas favorecen nombres de tipo tradicional mientras que las clases populares se decantan por nombres más populares o de moda. 


\section{Cambios de categoría, funcionalidad u oficialidad}

Encontramos en este apartado usos antroponímicos que proceden de sustantivos que pertenecen en principio a otras categorías nominales o a nombres que poseen otras aplicaciones o rango de oficialidad.

D1. El caso más paradigmático es lo que García Gallarín (2007a, 2007b) denomina nombres transparentes o parlantes, es decir, nombres comunes que pasan a convertirse en propios: Senda como nombre masculino; Rimas, Abril, Jade, Lluvia, Azul, Nirvana, Rubí, Nube, Senda, Alondra, Malva, Ámbar, Isla Sol, Luna Mar o Azalea como nombres femeninos. Una vez más comprobamos que esta tendencia afecta casi exclusivamente a los antropónimos femeninos. En ocasiones esta innovación se combina con los préstamos y da lugar a nombres tan singulares como Wisdom, Miracle, Godstime, Hug, Destiny y Iron para varón o Ebony, Harmony, Success y Favour para mujer. Este mismo proceso se ha llevado a cabo en otras lenguas peninsulares con nombres como eus. Iraultza 'revolución', cat. Bruc 'brezo’ o cat. Saüc 'saúco'.

D2. Algunos topónimos son empleados como antropónimos siguiendo una línea de atribución de reciente implantación en países como Estados Unidos y más asentada en lenguas como el vasco. Así, son nombres de varón Jericó, Saba, Itoitz y Sión, y nombres de mujer India, Itaca, Siena, Cíes, Noumidia, Paris, Savannah, Dakota, Izaro, Sinai, Kenya, Ankara o Namibia.

D3. En este mismo grupo incluimos dos tipos de elementos que modifican su empleo como nombres complementarios o familiares y se transforman en nombre oficial o parte de un nombre oficial. Eso ocurre con Júnior, pospuesto siempre a un nombre de varón como parte integrante oficial de su nombre y perdiendo su carácter potestativo de adyacente Uúnior Alexander, Jorge Júnior). También ganan oficialidad numerosos hipocorísticos, que funcionan autónomamente como nombres de persona, registrados ahora como oficiales, aunque su consideración inicial los sitúa en el nivel más coloquial de la denominación (Polge, 1983): Quique, Juanma, Curro, Nacho, Pepe, Edu, Kiko, Paco, Nando, Maisa, Lola, Maica, Mila o Álex.

\section{E. Variación ortográfica}

Uno de los recursos más empleados en la creación de nuevos nombres es a través de la variación ortográfica. Esta afecta sobre todo a nombres nuevos, procedentes de otras lenguas, para acomodarlos a la pronunciación española: Yeremy, Neizan, Brayan, Yonas, Estiven o Eimy. A veces presentan numerosas variantes gráficas: Maicol, Maikol, Maykol, Maykel, Eiden, Eyden, Eydan, Eidan, Aiden, Ayden o Ainhoa, Aynoha, Ahinoa, Ainoha, Ainoa. Otras veces las grafías ajenas a la forma original parecen responder a un intento de diferenciación del nombre, al uso de una grafía extranjera o quizás también a un desconocimiento de la forma establecida: Jhoan, Ulysses, Deyvid, Jhon, Jeyson, Patryk, Khloe, Mishelle, Shantal, Keisy, Sofiia, Mayka, Jhoselyn, Ekhiñe entre otros. 
Resulta sorprendente que este mecanismo, la creación ortográfica, esté adquiriendo un cierto uso en el ámbito onomástico cuando atenta contra un código que despierta actitudes conservadoras por parte de los hablantes 25 aunque en menor medida en el sistema onomástico, que ha sido más permisivo en la convivencia de variantes de un mismo étimo, aunque atenten en ocasiones con la unirreferencialidad que lo caracteriza (García Gallarín, 1998). Reyes y Marrero (2013) achacan este hecho a que los antropónimos se han situado en la periferia del sistema, "allí donde, por su lejanía y su singularidad, ya no puede llegar la norma con toda su contundencia puesto que se imponen, o lo impiden, causas ajenas a ella". Por esa misma razón, el hablante puede hacer un uso individual que no podría hacer con otros elementos del sistema, como los sustantivos comunes. Por su parte, García Gallarín (1998) atribuye la actual disparidad ortográfica en los antropónimos españoles a diversos factores: la dificultad de adaptar nombres procedentes de otras lenguas, entre las que se incluyen las otras peninsulares, y, en general, el desconocimiento de las reglas de transcripción de muchos de los nombres elegidos, sobre todo en algunos hablantes no cultos, que han optado por la adaptación de la versión oral de los diferentes términos frente a su forma escrita.

\subsection{La obsolescencia onomástica}

Para revisar los nombres cuyo uso está en retroceso acudiremos al mismo tipo de fuente que nos ha servido de punto de partida para el estudio de la innovación: las listas de los nombres de acuerdo con su edad media. En este caso, estudiaremos los nombres cuya edad media supera los 70 años. Como ocurría con el apartado anterior, no se trata de nombres que no sean compartidos por diferentes generaciones, sino cuyo perfil de usuario pertenece a la generación más anciana. Los datos globales de este conjunto de nombres los hemos detallado en la tabla 3.

\section{TABLA 3}

Detalle del corpus de nombres con edad media superior a 70 años

\begin{tabular}{lcc} 
& HOMBRES & MUJERES \\
Número total de nombres & 24.601 & 25.091 \\
\hline Nombres con edad media superior a 70 años & $131(0,5 \%)$ & $646(2,6 \%)$ \\
\hline Nombres compuestos & 34 & 73 \\
\hline Nombres con $\mathbf{n}^{\circ}$ de usuarios > 1000 & 1 & 92 \\
\hline Nombres extranjeros & 34 & 48 \\
\hline
\end{tabular}

25 Un buen ejemplo que sustenta la primera afirmación es el trabajo de Fauvet (2016) en Chile. 
Si comparamos la tabla 3 con la 2, observamos que este conjunto de nombres es muy inferior al de los jóvenes, que podríamos justificar por la diferencia en las cifras demográficas entre ambas generaciones, más numerosa en el caso de los jóvenes. La misma explicación podríamos atribuir a la mayor presencia de nombres femeninos en esta franja, ya que es sabido que las mujeres son más longevas que los varones y el saldo demográfico está a su favor. Sin embargo, las disparidades en los datos de nombres son mucho más marcadas de lo que harían suponer los datos demográficos. Así, hay proporcionalmente mayor número de nombres (casi 10 veces más) con edad media joven (11,4\%) que con edad media anciana $(1,6 \%)^{26}$. Igualmente hay proporcionalmente mayor número de nombres ( 5 veces más) de mujer (2,6\%) que de hombre $(0,5 \%)^{27}$. Esto nos remite a situaciones ya detectadas en trabajos anteriores (Fernández Juncal, 2019) en dos direcciones. Por una parte, se observa una tendencia en el repertorio general desde un sistema con número reducido de elementos, basados en procesos de herencia a un procedimiento de atribución fundamentado en la originalidad en la nomenclatura. En consonancia con esto, hay mayor abundancia de nombres nuevos en las generaciones más jóvenes y muchos menos y menos variedad en el grupo de edad mayor.

Por otra parte, esa propensión a la unicidad frente a la uniformidad es mucho más patente para las mujeres que para los hombres (Fernández Juncal, 2002 y 2019), por lo que hay también más nombres con el perfil de mujer anciana que con el perfil de hombre anciano.

Este conjunto de nombres se define por su carácter tradicional, adscrito mayoritariamente al santoral (Acindino, Afrodisia) y en menor medida al catálogo de advocaciones marianas (Veneranda) u otras festividades católicas (Patrocinio, Exaltación). Este tipo de antropónimos ha estado asociado al medio rural, donde han sido empleados de manera más general. Reyes y Marrero (2013) les adjudican, no sin cierta razón, el calificativo de estigmatizados. Hay un dato que refrenda el carácter anticuado y en vías de extinción de este tipo de nombres: apenas alguno de los que forman este conjunto aparece en el corpus de léxico disponible que elaboramos en Fernández Juncal y Hernández Muñoz (2019) al menos en las primeras 150 posiciones, lo que indica que están ya fuera del lexicón mental en el centro de interés o campo semántico de los nombres de persona.

Hay además dos tipos de elementos que también definen este subcorpus. En primer lugar, encontramos nombres que representan valores o ideales republicanos (Progreso (v), Concordia, Acracia, Fraternidad). Se trata de personas que, por edad, han nacido en la década de los 30 y, por lo tanto, han recibido su nombre en una etapa de laicismo. Como recuerda García Gallarín (2007b), fueron denominaciones que identificaron a los perdedores de la guerra civil

26 Mientras que la proporción entre las dos generaciones es de casi 6 a 4 a favor de la generación más joven.

27 Mientras que la proporción entre los dos sexos es de casi 6 a 4 a favor de las mujeres. 
y, por lo tanto, en muchas ocasiones se desestimaron y tuvieron que ser ocultados hasta la llegada de la democracia.

En segundo lugar, hallamos en nuestras listas nombres extranjeros, ingleses y alemanes y, en menor medida, escandinavos y franceses. Como es hábito en esos países, se presentan casi siempre como nombres compuestos o múltiples (Trevor William, Friedrich Wilhelm, Brenda Joyce, Lieselotte, Jytte, Christiane Marie). Estas denominaciones son el reflejo de un tipo de inmigración distinta a la que se detectaba en el subcorpus joven: la de los jubilados extranjeros residentes en España. De esta forma se ven a través de los registros onomásticos las diferentes procedencias y características de los movimientos poblacionales.

Para finalizar aportamos en las tablas 4a y 4b la comparativa de los primeros 25 puestos de tres grupos de nombres diferentes: los nombres con edad media más baja, los nombres con edad media más elevada y los nombres más frecuentes de residentes en España en su conjunto y para los nacidos en las últimas décadas. Basta un examen somero para detectar los cambios en las tendencias, los gustos y los diferentes sistemas de atribución que se han ido sucediendo a lo largo del periodo estudiado.

\section{TABLA 4A}

Nombres de varón con edad media menor, con edad media mayor y más frecuentes en el repertorio en conjunto y entre los recién nacidos entre 2002 y 2016

\begin{tabular}{|c|c|c|c|c|c|c|}
\hline $\begin{array}{l}\text { NOMBRES CON } \\
\text { EDAD MEDIA } \\
\text { MÁS BAJA }\end{array}$ & $\begin{array}{l}\text { EDAD } \\
\text { MEDIA }\end{array}$ & $\begin{array}{l}\text { NOMBRES CON } \\
\text { EDAD MEDIA } \\
\text { MÁS ALTA }\end{array}$ & $\begin{array}{l}\text { EDAD } \\
\text { MEDIA }\end{array}$ & $\begin{array}{l}\text { NOMBRES MÁS } \\
\text { FRECUENTES EN } \\
\text { EL REPERTORIO }\end{array}$ & $\begin{array}{l}\text { EDAD } \\
\text { MEDIA }\end{array}$ & $\begin{array}{l}\text { NOMBRES } \\
\text { FRECUENTES } \\
2002-16\end{array}$ \\
\hline Aylan & 2,0 & Progreso & 78,3 & Antonio & 55,5 & Alejandro \\
\hline Keylor & 2,2 & Acindino & 77,9 & José & 60,8 & Daniel \\
\hline Eiden & 2,6 & Frumencio & 77,8 & Manuel & 54,6 & Pablo \\
\hline Eyden & 2,6 & Auxilio & 77,4 & Francisco & 56,9 & David \\
\hline Bruc & 2,7 & Frederick George & 76,8 & David & 29,7 & Hugo \\
\hline Yeiden & 2,7 & Vitores & 75,3 & Juan & 55,4 & Adrián \\
\hline Tahiel & 2,8 & Maturino & 75,3 & José Antonio & 48,8 & Álvaro \\
\hline Jeyden & 2,8 & Daciano & 75,2 & Javier & 32,3 & Javier \\
\hline Ianis Nicolas & 2,8 & Auspicio & 74,8 & José Luis & 52,5 & Diego \\
\hline Irai & 2,9 & Abundio & 74,4 & Daniel & 26,5 & Sergio \\
\hline Neymar & 2,9 & Valerico & 74,4 & Francisco Javier & 43,8 & Mario \\
\hline Yanis Andrei & 2,9 & Hilarino & 74,0 & Jesús & 48,0 & Manuel \\
\hline Neyzan & 3,1 & Teótimo & 73,9 & Carlos & 38,3 & Marcos \\
\hline Thiago Alexander & 3,1 & Adoración & 73,9 & Alejandro & 25,0 & Carlos \\
\hline
\end{tabular}




\begin{tabular}{lllllll}
\hline Lyan & 3,1 & Ronald Charles & 73,8 & Miguel & 46,2 & Jorge \\
\hline Edahi & 3,2 & Pilar & 73,7 & José Manuel & 46,4 & Iván \\
\hline Thiago David & 3,2 & Euquerio & 73,7 & Rafael & 50,2 & Miguel \\
\hline Lucas Mihai & 3,2 & Floreal & 73,5 & Pedro & 53,1 & Lucas \\
\hline Neizan & 3,3 & Kenneth George & 73,5 & Miguel Ángel & 42,8 & Iker \\
\hline Barae & 3,3 & Exuperio & 73,4 & Ángel & 49,9 & Antonio \\
\hline
\end{tabular}

\section{TABLA 4B}

Nombres de mujer con edad media menor, con edad media mayor y más frecuentes en el repertorio en conjunto y entre los recién nacidos entre 2002 y 2016

\begin{tabular}{|c|c|c|c|c|c|c|}
\hline $\begin{array}{l}\text { NOMBRES CON } \\
\text { EDAD MEDIA } \\
\text { MÁS BAJA }\end{array}$ & $\begin{array}{l}\text { EDAD } \\
\text { MEDIA }\end{array}$ & $\begin{array}{l}\text { NOMBRES CON } \\
\text { EDAD MEDIA } \\
\text { MÁS ALTA }\end{array}$ & $\begin{array}{l}\text { EDAD } \\
\text { MEDIA }\end{array}$ & $\begin{array}{l}\text { NOMBRES MÁS } \\
\text { FRECUENTES }\end{array}$ & $\begin{array}{l}\text { EDAD } \\
\text { MEDIA }\end{array}$ & $\begin{array}{l}\text { NOMBRES } \\
\text { FRECUENTES } \\
2002-16\end{array}$ \\
\hline Cataleya & 1,6 & Afrodisia & 82,9 & María Carmen & 56,2 & Lucía \\
\hline Cattleya & 1,6 & Segismunda & 81,2 & María & 48,7 & María \\
\hline Sarayma & 1,9 & Parmenia & 80,4 & Carmen & 60,4 & Paula \\
\hline Ritaj & 2,1 & Acracia & 80,3 & Josefa & 67,5 & Sara \\
\hline Retaj & 2,1 & Fraternidad & 80,0 & Ana María & 50,4 & Alba \\
\hline Daenerys & 2,2 & Ermerinda & 79,7 & Isabel & 57,0 & Laura \\
\hline Xuhare & 2,2 & Serviliana & 79,6 & María Pilar & 56,2 & Carla \\
\hline Chloe Valentina & 2,3 & Domina & 79,5 & María Dolores & 55,9 & Marta \\
\hline Sofia Andreea & 2,3 & Capitulina & 79,5 & Laura & 27,6 & Claudia \\
\hline Arya & 2,4 & Procopia & 79,4 & María Teresa & 56,3 & Sofía \\
\hline Rinad & 2,4 & Iluminación & 79,2 & Ana & 43,6 & Daniela \\
\hline Khloe & 2,4 & Exiquia & 79,1 & Cristina & 32,9 & Julia \\
\hline Naevia & 2,4 & Sancho Abarca & 79,1 & María Ángeles & 54,6 & Irene \\
\hline Sofia Ioana & 2,4 & Eustasia & 79,0 & Marta & 28,5 & Martina \\
\hline Eshaal & 2,4 & Teódula & 79,0 & Francisca & 64,4 & Andrea \\
\hline Assinat & 2,7 & Predestina & 78,9 & Antonia & 64,2 & Ana \\
\hline Roaya & 2,8 & Luftolde & 78,9 & María Isabel & 51,9 & Carmen \\
\hline Zhoe & 2,8 & Crescenciana & 78,8 & María José & 45,2 & Elena \\
\hline Anabia & 2,8 & Dativa & 78,7 & Dolores & 66,5 & Nerea \\
\hline Maya Andreea & 2,9 & Abundia & 78,5 & Lucia & 23,0 & Valeria \\
\hline
\end{tabular}




\section{Conclusiones}

En el presente artículo hemos podido analizar cambios lingüísticos en tiempo aparente en el ámbito onomástico. A partir de los datos proporcionados por el INE hemos examinado diferentes tendencias antroponímicas en diferentes épocas de nuestro país. Nos hemos detenido en primer lugar en los nombres permanentes, aquellos muy instalados en el catálogo nominal español, que han permanecido en posiciones elevadas de popularidad en todas las décadas estudiadas. Se trata de términos que, además de frecuentes, son parte del acervo distintivo e identitario español y que también se repiten en otros repertorios a los que hemos tenido acceso, especialmente el que se deduce de un estudio de disponibilidad léxica (Fernández Juncal y Hernández Muñoz, 2019), que permite acceder al lexicón mental que los hablantes activan ante determinados estímulos de carácter conceptual y que nos permiten detectar estereotipos asociados a un campo nocional.

En segundo lugar, hemos indagado en los procesos de renovación e innovación onomástica que se han llevado a cabo en las últimas décadas. Tanto en el corpus completo de nombres (con frecuencia superior a 20) ordenado por edad media de sus usuarios como en el corpus de los nombres más habituales entre los nacidos desde 2002 se observa de manera muy destacada una ampliación del repertorio, que implica mayor diversificación en la distribución de nombres, con frecuencias menos agrupadas que en épocas anteriores, en las que pocos nombres concentraban la denominación de un buen número de portadores.

Este proceso de innovación onomástica se realiza mediante diversos mecanismos que hemos agrupado en cinco grandes bloques. El principal recurso de cambio es la importación de antropónimos de otras lenguas, nacionales o extranjeras, hecho favorecido por una legislación más permisiva y estimulado por varios factores coincidentes, que confluyen en uno, los desplazamientos humanos en todo el mundo: por una parte, una inmigración de cuño joven procedente de países subdesarrollados o en vías de desarrollo, tanto hispanófonos como hablantes de otras lenguas. En el caso de los primeros su tradición onomástica ya estaba muy impregnada por una nomenclatura con préstamos y creaciones extranjerizantes. Por otra parte, la globalización y la urbanización (Gerritzen, 2006) también redundan en la expansión de modas y novedades en un ámbito internacional. Otros mecanismos de innovación tienen que ver precisamente con la incorporación de nombres que están asociados a acontecimientos y circunstancias de la época (personas famosas, personajes de ficción, etc.) o con la recuperación de nombres de tradición clásica que habían perdido su uso o no estaban plenamente integrados en las costumbres onomásticas.

El empleo de nombres compuestos o múltiples es una constante que viene de antiguo. En la actualidad siguen teniendo un gran peso dentro del conjunto de nombres registrados, pero con la diferencia de que ha descendido muy significativamente la utilización de nombres dobles establecidos de manera consuetudinaria a favor de nombres yuxtapuestos, creaciones 
ad hoc por la suma de unidades autónomas. No obstante, este empleo muy probablemente no tiene su traslación al uso del nombre completo en la vida cotidiana. Hay otros dos mecanismos que tienen que ver con la transformación del repertorio existente. Por una parte, la variación ortográfica ofrece oportunidades de diferenciación en nombres muy similares aunque violenta en muchas ocasiones el tradicional sistema de trasliteración del español, dificultando, por ende, la correcta escritura del término tal como está registrado. Por otra parte, diferentes sustantivos cambian su categoría, función y rango para incorporarse al elenco antroponímico: nombres comunes se transforman en propios en un proceso inverso a la creación de genéricos o términos deonomásticos. Lo mismo ocurre con los topónimos, que trasladan y amplían su área de denominación, y los hipocorísticos, que salen del ámbito coloquial y doméstico y adquieren condición de oficialidad.

Todos estos recursos de ampliación y renovación del catálogo no se producen con la misma intensidad en todos los colectivos. La búsqueda de originalidad y novedad es más perceptible en la atribución onomástica de mujeres, beneficiarias en mayor medida de los procedimientos de innovación.

Abordamos finalmente los nombres obsolescentes, generalmente de origen religioso, con estereotipo de carácter rural. Estos nombres nos permiten confrontar los cambios en gustos y tendencias que se han producido en varias generaciones cuando los observamos en paralelo a los más recientes, de muy diferente origen y naturaleza. Además el contraste de épocas nos permite ver la confluencia en una misma sincronía de diferentes movimientos demográficos de inmigración.

\section{Bibliografía citada}

Ainiala, Teri, y Jan-Ola Östman, 2017: "Introduction: Socio-onomastics and Pragmatics" en Teri Ainiala y Jan-Ola Östman (eds): Socio-onomastics, Philadelphia: John Benjamins, 1-18.

AldRIN, Emilia, 2016: "Names and Identity" en Carole Hough (ed.): The Oxford Handbook of Names and Naming, Oxford: Oxford University Press, 382-394.

ALDRIN, Emilia, 2017: "Creating identities through the choice of first names" en Teri AInIALA y JanOla Östman (eds): Socio-onomastics, Philadelphia: John Benjamins, 45-68.

Bajo Pérez, Elena, 2002: La caracterización morfosintáctica del nombre propio, A Coruña: Toxosoutos.

BLoothooft, Gerrit, y Kees MANDEMAKERS, 2014: "Exploring co-variation in the (historical) Dutch civil registration" en Actes deI XXIV Congrés Internacional d'ICOS sobre Ciències Onomàstiques, Barcelona: Generalitat de Catalunya, 271-282. 
BramwelL, Ellen, 2014: "Personal Naming and Society: A comparative study of disparate communities" en Actes del XXIV Congrés Internacional d'ICOS sobre Ciències Onomàstiques, Barcelona: Generalitat de Catalunya, 712-718.

Bullet, Richard, 1978: "First Names and Political Change in Modern Turkey", International Journal of Middle East Studies 9 (4), 489-495.

Bush, Stephen, Anna Powell-Smith y Tom Freeman, 2018: "Network analysis of the social and demographic influences on name choice within the UK (1838-2016)", PLOS ONE 13 (10), e02057.

Cid Abasolo, Karlos, 2007: "Nombres vascos en Madrid" en Consuelo Garcia Gallarin (coord.): Los nombres del Madrid multicultural, Madrid: Ediciones Parthenon, 65-98.

Colman, Andrew, Wladyslaw Sluckin y David Hargreaves, 1981: "The effect of familiarity on preferences for surnames", British Journal of Psychology 72, 363-369.

Fauvet, Marion, 2016: Actitudes e ideologías de hispanohablantes de Santiago de Chile acerca de la ortografía. Tesis de pregrado, Universidad de Chile.

Fernandez Juncal, Carmen, 2002: "Algunos datos socionomásticos de una comunidad de la región funcional de Salamanca" en J. A. BARToL y otros (eds.): Nuevas aportaciones al estudio de la lengua española. Investigaciones filológicas, Salamanca: Luso-Española de Ediciones, 257-264.

Fernandez Juncal, Carmen, 2009: "Patrones sociolingüísticos de la onomástica", Revista de Lingüistica Española 38 (2), 5-20.

Fernandez Juncal, Carmen, 2011: "El sistema antroponímico como diasistema”, Nouvelle Revue d'Onomastique 53, 143-152.

FernANDEZ JUnCAL, Carmen, 2018: "Evolution of Anthroponyms in an Area of Linguistic Transition: a Socio-Onomastic Study”, Names: A Journal of Onomastics 66 (2), 85-95.

Fernandez Juncal, Carmen, 2019: "Evolución de los usos antroponímicos en España”, Moenia 25, $149-177$.

Fernandez Juncal, Carmen, y Natividad Hernández Muñoz, 2019: "Disponibilidad léxica y socionomástica", OGIGIA 25, 185-210.

Fernandez Leborans, M. ${ }^{a}$ Jesús, 1999: “El nombre propio" en Ignacio Bosque y Violeta Demonte (eds.): Gramática descriptiva de la lengua española, I. I Sintaxis básica de las clases de palabras, Madrid: Espasa, 77-128. 
Fernandez Pérez, Enrique, 2015: El nombre y los apellidos. Su regulación en derecho español y comparado. Tesis doctoral, Universidad de Sevilla.

FRAl, Patricia, 2016: "Sócio-onomástica: Uma nona abordagem metodológica", Entreletras 7 (1), 91-106.

Garcia Gallarín, Consuelo, 1997: Nombres del siglo XX. Contribución al estudio de la antroponimia madrileña, Madrid: Universidad Complutense de Madrid.

García Gallarín, Consuelo, 1998: Los nombres de pila españoles, Madrid: Ediciones del Prado.

García Gallarin, Consuelo, 2007a: "La evolución de la antroponimia hispanoamericana" en Consuelo García Gallarín (ed.): Los nombres del Madrid multicultural, Madrid: Parthenon, 209-234.

García Gallarín, Consuelo, 2007b: “Tradición e innovación antroponímicas (Madrid, 1996-2006)” en Consuelo García Gallarín (ed.): Los nombres del Madrid multicultural, Madrid: Parthenon, 99-134.

Garcia Gallarín, Consuelo, 2009: "Variación y cambio antroponímicos” en Consuelo Garcia GallaRín y Karlos Cid (eds.): Los nombres de persona en la sociedad y en la literatura de tres culturas, Madrid: Sílex, 71-110.

Garcia Gallarin, Consuelo, 2010: "Rutas de la antroponimia hispánica” en Carmen Maiz (ed.): Nombre propio e identidad cultural, Madrid: Sílex, 57-99.

Garcia Gallarin, Consuelo, 2014a: Diccionario histórico de nombres de América y España. Estudio preliminar, Madrid: Silex.

Garcia Gallarín, Consuelo, 2014b: "Nombre propio y ambigüedad" en Juan Ortega y Victoria Navas (coords.): Del nombre oficial al nombre familiar, Madrid: Orto, 43-72.

Gary PrIeUR, Marie-Noëlle, 1994: Grammaire du nom propre, París: PUF, Linguistique Nouvelle.

GeRRITZEn, Doreen, 2006: “Naming Children in a Globalizing World”, Acta Onomastica XLVII, 177-184.

Guérios, Mansur, 1981: Dicionário Etimológico de nomes e sobrenomes, São Paulo: Editora Ave Maria.

Hough, Carole, 2016: "Introduction" en Carol Hough (ed.): The Oxford Handbook of Names and Naming, Oxford: OUP, 1-13.

Jiménez Segura, Selene, 2014: "Los procesos de cambio de los modelos de atribución antroponímica tradicional y a partir de la moda en el municipio de Tlalnepantla de Baz, Estado de México. Tres calas: 1930, 1960 y 1990", Trama 10 (20), 127-148. 
Kessler, David, Yosef Maruvka, Joergen Ouren y Nadav Shnerb, 2012: "You Name It - How Memory and Delay Govern First Name Dynamics", PLOS ONE 7 (6).

Lieberson, Stanley, 2000: A Matter of Taste. How Names, Fashions, and Culture Change, New Haven: Yale University Press.

López Franco, Yolanda, 2010: Un siglo de nombres de pila en Tlalnepantla de Baz, México, D.F.: Editorial Plaza y Valdés.

López Franco, Yolanda, 2014: “Comparaison des prénoms attribués en 1970 et 1975 dans deux comunes romanophones. Une approche socioanthroponymique" en Actes del XXIV Congrés Internacional d'ICOS sobre Ciències Onomàstiques, Barcelona: Generalitat de Catalunya, 821-832.

Mateos, Pablo, y Ken Tucker, 2008: “Forenames and Surnames in Spain in 2004", Names 56 (3), 165-184.

Polge, Henri, 1983: “Hypocoristiques et antiphonétique”, Nouvelle Revue d'Onomastique 2, 119-120.

Reyes Díaz, Josefa, y Vicente Marrero, 2013: "Antropónimos. Análisis de una muestra canaria de los dos últimos decenios”, Nouvelle Revue d’Onomastique 55, 191-231.

Rossı, Alice, 1965: "Naming Children in Middle-Class Families", American Sociological Review 30, 499-513.

Selde, Márcia, 2016: “Métodos de pesquisa em Antroponomástica”, Domínios de Lingu@gem / Uberlândia 10 (3), 1146-1171.

Seide, Márcia, y Tatiana Grespan, 2013: "Mais Wellicas Samaras e Hendryas Nicoles, menos Marias Luísas e Joões Pedros: os novos nomes duplos do sistema antroponímico brasileiro", Recorte 10 (2), 1-19.

Thonus, Terese, 1992: "Anderson, Maicon and Thiago: English names in Brazil”, American Speech $67(2), 175-189$.

Vandebosch, H., 1998: “The Influence of Media on Given Names”, Names 46, 243-262.

Weitman, Sasha, 1981: "Some Methodological Issues in Quantitative Onomastics", Names 29 (3), 181-196.

Wheeler, Sara, 2018: "Autoethnographic onomastics: Transdisciplinary scholarship of personal names and 'our-stories'”, Methodological Innovations 2018, 1-11. 